\title{
Duloxetine for the treatment of generalized anxiety disorder: a review
}

\author{
Ahsan Y Khan \\ Matthew Macaluso \\ Department of Psychiatry \\ and Behavioral Sciences, University \\ of Kansas School of Medicine-Wichita, \\ Wichita, KS, USA
}

\begin{abstract}
Approximately 16 million people in the United States suffer from anxiety disorders alone, while another 12 million experience both anxiety and at least one other psychiatric condition. Generalized anxiety disorder (GAD) has lifetime prevalence rates between $5 \%$ and $6 \%$. Treatment of GAD is aimed primarily at symptom reduction. Duloxetine, a serotonin norepinephrine reuptake inhibitor (SNRI), received Food and Drug Administration (FDA) approval for the treatment of GAD in 2007. This article reviews the pharmacologic profile and seminal clinical trials associated with the FDA indication of duloxetine for GAD. A literature search performed using PubMed with the keywords "duloxetine", "gad", "generalized anxiety disorder", and "venlafaxine XR" yielded 27 articles. We also focused on papers that pooled data from these seminal studies. Data on file from Eli Lilly were also reviewed, including data from the Eli Lilly website. Based on this search, duloxetine was found to be an FDA-approved treatment option for GAD that has been studied in several double-blind, placebo-controlled clinical trials. This review of duloxetine will help physicians to interpret clinical studies properly and also help them to make an informed decision about which patients are the most appropriate candidates for a trial of duloxetine.
\end{abstract}

Keywords: duloxetine, generalized anxiety disorder, clinical trials, serotonin norepinephrine reuptake inhibitor (SNRI)

\section{Introduction}

Anxiety disorders were classified as neurotic disorders until the establishment of the DSM-III in 1980. Approximately 16 million people in the United States suffer from anxiety disorders alone, while another 12 million experience both anxiety and at least one other psychiatric disorder. ${ }^{1}$

Generalized anxiety disorder (GAD) involves excessive worry about everyday life events, on more days than not, for a period of at least 6 months. ${ }^{2}$ According to DSM-IV-TR, patients must find it "difficult to control the worry" and must present with 3 of the following 6 criteria: restlessness or feeling keyed up, being easily fatigued, difficulty concentrating or mind going blank, irritability, muscle tension, or sleep disturbance. ${ }^{2}$ Patients with GAD often present hyper-vigilant, with somatic complaints such as musculoskeletal pain or gastrointestinal disturbance. ${ }^{3}$ GAD has lifetime prevalence rates between $5 \%$ and $6 \%$, and a male to female ratio of $1: 2 .{ }^{4}$

Treatment of GAD is aimed at symptom reduction, often utilizing selective serotonin reuptake inhibitors (SSRIs), serotonin and norepinephrine reuptake inhibitors (SNRIs), buspirone, benzodiazepines and/or psychotherapy. In 1991, Butler et al published evidence supporting the use of cognitive behavioral therapy (CBT) in the treatment of GAD. ${ }^{5}$ Both venlafaxine and duloxetine have been Food and Drug Administration (FDA) approved for the treatment of GAD.

Another SNRI, milnacipran, is not yet available in the United States, but is utilized clinically to treat major depressive disorder (MDD) and chronic pain in other parts of the world including Europe. ${ }^{6}$ Duloxetine, an SNRI, received FDA approval for MDD
Correspondence: Ahsan Y Khan, MD Department of Psychiatry and Behavioral Sciences, University of Kansas School of Medicine-Wichita, 1010 N. Kansas Street,Wichita, KS 67214, USA

Email akhan@kumc.edu 
and Diabetic peripheral neuropathic pain (DPNP) in $2004 .^{7}$ Preclinical studies of duloxetine indicated an anxiolytic effect based on reduction in animal anxiety behaviors. ${ }^{7,8}$ Finally, duloxetine received FDA approval for the treatment of GAD in February of 2007.7 According to Eli Lilly (the manufacturer of duloxetine), GAD patients treated with duloxetine in clinical trials showed a $46 \%$ improvement in anxiety symptoms when compared with placebo. ${ }^{8}$

This article will review the pharmacologic profile and seminal clinical trials associated with the FDA indication of duloxetine for GAD. Confidence in the predictability of safety, tolerability and efficacy of a medication is directly proportional to the magnitude and quality of its published trials. The goal of this paper is to assess the amount of information from wellcontrolled systematic trials. This information forms the basis for evidence-based medical practice and helps clinicians to make the most appropriate decisions.

\section{Materials and methods}

A literature search was performed using PubMed with keywords "duloxetine," "gad," "generalized anxiety disorder," and "venlafaxine XR." The search yielded 27 articles. We included large, multi-center studies and focused on the seminal papers that led to the FDA approval for duloxetine in GAD. We also focused on papers that pooled data from these seminal studies.

Data on file from Eli Lilly was also reviewed, including data from the Eli Lilly website. We also included information available in the duloxetine package insert, as well as the Physicians Desk Reference (PDR).

Before we examine data on the efficacy of duloxetine as a treatment for GAD, we review the basic pharmacology of duloxetine.

\section{Pharmacodynamics of duloxetine}

The ratio of duloxetine's affinity constants for serotonin (5HT) and norepinephrine (NE) reuptake pumps is closer to 1, meaning it is a more balanced and potent inhibitor for these two reuptake pumps than venlafaxine. ${ }^{9}$ Duloxetine has a $\mathrm{Ki}$ of $0.8 \mathrm{nM}$ for $\mathrm{NE}$ and $7.5 \mathrm{nM}$ for 5HT, whereas venlafaxine's Ki for NE and 5HT are $2480 \mathrm{nM}$ and $82 \mathrm{nM}$ respectively. ${ }^{9}$ Hence, duloxetine has greater affinity for both $5 \mathrm{HT}$ and NE. ${ }^{10}$ Like venlafaxine and milnacipran, duloxetine has minimal affinity for dopamine, histamine (H1), adrenergic, muscurinic, opiate, gamma amino butyric acid (GABA), and substance P receptors. ${ }^{9}$

\section{Pharmacokinetics of duloxetine}

Compared with most antidepressants, duloxetine exhibits some unusual absorption properties. Duloxetine is rapidly hydrolyzed in acidic media to naphthol, which has no antidepressant activity. ${ }^{10}$ To overcome this issue, the pellets inside the duloxetine capsule are enteric coated, but the capsule is not. This enteric coating of the pellets resists dissolution until pellets reach a segment of the gastrointestinal tract where the $\mathrm{pH}$ is favorable. This explains why the maximal plasma concentration $\left(\mathrm{C}_{\max }\right)$ of duloxetine usually does not occur until 6 hours post dose. ${ }^{10}$

Duloxetine has a mean plasma half-life of 12 hours, but it can be dosed once daily as the central nervous system half-life may be very different from the plasma half-life. In one reported study, duloxetine dosed at 20 to $40 \mathrm{mg}$ twice daily in 12 healthy male volunteers exhibited linear pharmacokinetics with steady state plasma concentrations typically reached within 3 days of stable dosing. ${ }^{9}$ Duloxetine is highly protein bound (96\%), primarily to albumin and alpha-1-acid glycoprotein. ${ }^{10}$

Duloxetine is metabolized by CYP-2D6 and 1A2 isoenzymes with no active metabolites. ${ }^{10}$ Because of CYP-1A2 metabolism, duloxetine use in smokers results in a one third reduction in bioavailability compared with non-smokers. ${ }^{10}$ However, there is no clinical recommendation for a dosage adjustment based on smoking status. ${ }^{10}$ Duloxetine should not be used with CYP-1A2 inhibitors such as fluvoxamine. ${ }^{9}$

Based on in vitro studies, duloxetine is a more potent inhibitor of CYP 2D6 than CYP 1A2. Concomitant use of duloxetine at a dose of $120 \mathrm{mg}$ /day increased levels of desipramine 3-fold, a model CYP 2D6 substrate, indicating substantial inhibition of CYP 2D6. ${ }^{11}$ However, the recommended dose of duloxetine for the treatment of MDD is 40 to $60 \mathrm{mg} /$ day. At these recommended doses, the increase in plasma levels of CYP 2D6 substrates drugs like desipramine would be expected to be one third to one half that seen with the $120 \mathrm{mg} /$ day dose (ie, 1 to 1.5 fold increase). This is consistent with the linear pharmacokinetics of duloxetine over this dose range, meaning it has no effect on its own CYP 2D6 mediated clearance. ${ }^{11}$ The effect of duloxetine on CYP 2D6 is significantly less than as seen with fluoxetine or paroxetine at their lowest effective antidepressant doses and approximately the same as seen with escitalopram and citalopram. ${ }^{9}$ Thus duloxetine should be used with caution when combined with potent inhibitors of CYP-2D6 such as paroxetine and drugs using CYP 2D6 for their own clearance, particularly those with narrow therapeutic indexes, as significantly high concentrations of duloxetine may result. ${ }^{10}$

Duloxetine is excreted in urine (70\%) and feces (20\%), with less than $1 \%$ excreted unchanged (urine). ${ }^{12}$ 


\section{Dosing}

Clinical trials studying the efficacy of duloxetine in the treatment of GAD utilized doses ranging from $60 \mathrm{mg}$ to $120 \mathrm{mg}$ daily. ${ }^{3,13,14}$ Duloxetine is available in capsules of $20 \mathrm{mg}, 30 \mathrm{mg}$, and $60 \mathrm{mg}$ strengths. According to the manufacturer, the recommended dose of duloxetine in GAD is $60 \mathrm{mg} /$ day. ${ }^{7}$ The manufacturer states: "There is no evidence that doses greater than $60 \mathrm{mg}$ /day confers additional benefit, while adverse reactions such as dizziness, fatigue, somnolence, constipation, and decreased appetite were observed to be dose-dependent."

\section{Safety}

Pooled data from clinical trials involved four cases of overdose ingesting up to $1400 \mathrm{mg}$ of duloxetine, with no reported fatalities. For the purposes of the studies, overdose was defined as dose $>240 \mathrm{mg}$. Cardiovascular adverse events and toxicity in overdose associated with venlafaxine do not seem to be a concern with duloxetine. ${ }^{14}$

Duloxetine is not recommended for patients with end stage renal disease (ESRD), because following a single dose of 60 $\mathrm{mg}$ of duloxetine, the elimination half-life remains the same, but the $\mathrm{C}_{\text {max }}$ and AUC (area under the curve used to estimate bioavailability) values are approximately $100 \%$ greater compared with individuals with normal renal function. ${ }^{15}$

Because hepatic insufficiency impairs duloxetine metabolism, some researchers have suggested that such patients not be prescribed duloxetine. ${ }^{13}$ After a single dose of $20 \mathrm{mg}$, the half-life of duloxetine was 3 times longer, the mean AUC was 5 times greater, and the plasma clearance was 6 to 7 times less in 6 cirrhotic patients with moderate liver impairment versus what was seen in age and gender matched healthy controls. ${ }^{9}$

Preclinical studies demonstrated the potential for substantial central pharmacodynamic drug-drug interactions when duloxetine as well as other 5HT and NE uptake inhibitors are coadministered with drugs having specific effects on central 5HT, NE, and DA mechanisms. ${ }^{9}$ Such 5HT and NE reuptake pump inhibitors include tertiary and secondary amine tricyclic antidepressants (TCAs), selective 5HT uptake inhibitors (SSRIs), selective NE uptake pump inhibitors (eg, reboxetine), and other dual 5HT and NE uptake pump inhibitors (eg, venlafaxine and milnacipran). For similar reasons, the duloxetine package insert includes a warning against using duloxetine in combination with monoamine oxidase inhibitors (MAOIs). ${ }^{7}$ Such a combination has the potential to cause both a serotonin syndrome, as can occur with simultaneous administration of an SSRI and a MAOI, and a hypertensive crisis, as can occur with the combined administration of an selective NE reuptake pump inhibitor (eg, desipramine or reboxetine) and a MAOI. ${ }^{7}$

Duloxetine is currently pregnancy category $\mathrm{C}$ and possible effects on labor, delivery, and a developing fetus are unknown at this time. ${ }^{10}$ Use of duloxetine during pregnancy should be supervised by an expert, only after careful assessment of risk, patient education, and informed consent. ${ }^{10}$

Data from 4 randomized, double-blind, placebo controlled trials of duloxetine in patients $\geq 65$ years of age showed it to be a safe treatment modality in elderly with GAD. The study showed a high rate of discontinuation (22.2\%) due to adverse effects. ${ }^{16}$

\section{Adverse effects}

Patients should be made aware of the risks, benefits, possible side effects and alternatives associated with any medication prior to treatment. Pooled data from 3 clinical trials revealed a $16 \%$ discontinuation rate for patients treated with duloxetine compared with only $4 \%$ for placebo. ${ }^{8}$ The venlafaxine comparator arm of one study showed only an $11 \%$ discontinuation rate, which was lower than the $23 \%$ discontinuation rate shown in previous pooled data. ${ }^{14}$ The most common adverse effects included nausea, fatigue, dry mouth, somnolence, vomiting, insomnia, and sexual side effects. ${ }^{8}$

Side effects commonly associated with duloxetine are similar to those seen with venlafaxine or SSRIs. Nausea and insomnia are common side effects of duloxetine, although nausea seems to be less common than with SSRIs. After the first week of treatment, nausea rates associated with duloxetine are comparable to placebo. ${ }^{13}$ PDR indicates a nausea rate of $31 \%$ with venlafaxine, compared to $22 \%$ with duloxetine, at 75 to $225 \mathrm{mg}$ and 40 to $120 \mathrm{mg}$ respectively. ${ }^{12}$

Eight- to nine-week clinical trials studying duloxetine use in depression resulted in a $1.1 \mathrm{lb}(1 \mathrm{lb}=0.45 \mathrm{~kg})$ weight loss in the treatment group compared to a $0.5 \mathrm{lb}$ weight gain in the placebo group. ${ }^{3,13,14}$ One year follow-up data showed a weight gain of $2.46 \mathrm{lb}$ for patients in the treatment group, but no placebo group data were available. ${ }^{10}$

Clinical trial data showed clinically insignificant elevations in liver function tests in some patients, with most experts recommending that duloxetine not be used in those with a history of severe liver abnormalities. On follow-up, abnormalities in liver function tests were noted to decrease, regardless of whether or not the patient stopped using duloxetine. ${ }^{3}$

Sexual side effects were measured prospectively utilizing the Arizona Sexual Experience Scale (ASEX). Short-term clinical trials showed statistically significant sexual side 
effects for duloxetine when compared to placebo $(p=0.007)$. Male anorgasmia was the main effect noted. However, after 6 months the treatment group showed no statistically significant increase in sexual side effects compared with the placebo group $(\mathrm{p}=0.677) .{ }^{17}$

Urinary retention or hesitancy was rarely reported in clinical trials. Less than $2 \%$ of patients encountered this side effect, and no patient was catheterized. ${ }^{18}$ Clinical trials showed no evidence of QT prolongation, effect on glucose metabolism, hematologic abnormalities or difficulties with other organ systems. ${ }^{13}$

There were no reported cases of mania in patients taking duloxetine, compared with $2 \%$ of placebo controls. As with SSRIs and venlafaxine, patients taking duloxetine should be carefully monitored for treatment-emergent mania. Concomitant use of a mood-stabilizing medication should be considered in such cases. ${ }^{10}$

Sudden discontinuation can result in withdrawal symptoms, including but not limited to dizziness, anxiety, nausea, and headache. Because of possible discontinuation syndrome, duloxetine should be tapered rather than stopped abruptly. ${ }^{14}$ Patients should be made aware of the discontinue syndrome at the start of treatment with duloxetine.

However, the above clinical trials showed that "discontinuation-emergent adverse events" for duloxetine were statistically significant comparable to placebo. ${ }^{13,14}$ The venlafaxine comparator arm of study 3 also showed statistically significant "discontinuation-emergent adverse events" compared with placebo. ${ }^{14}$

In May of 2007 the FDA expanded its black box warning regarding antidepressant medication and suicidality to include young adults aged 18 to 24 years. A case series by Parikh et al showed a close relationship between suicidality and duloxetine use. In the cases presented, suicidal ideation was linked to an increase in duloxetine dose. ${ }^{19}$ In all cases presented, patients were no longer suicidal upon discontinuation of duloxetine. All patients prescribed antidepressant medication should provide appropriate informed consent on the risk of suicidality prior to the start of treatment.

Having reviewed the basic pharmacology and safety data on duloxetine, we will now look at the bedrock studies that led to FDA approval for the treatment of GAD.

\section{Efficacy in GAD}

We will discuss data from clinical trials that were designed to assess the efficacy of duloxetine in treating patient's meeting DSM-IV-TR criteria for GAD.,33,14 Each study utilized the Hamilton Rating Scale for Anxiety (HAM-A) score as the primary efficacy measure. Secondary efficacy measures included HAM-A items for tension and anxious mood, HAM-A Psychic and Somatic Factors, Hospital Anxiety and Depression Scale (HADS), Clinical Global Impressions Improvement scale (CGI-I) scores, and Patient Global Impression-Improvement Scale (PGI-I). ${ }^{3,13,14}$

The first study was a 9-week, double-blind, placebo controlled trial of 513 patients meeting DSM-IV-TR criteria for GAD. ${ }^{3}$ Inclusion criteria included age $>18$ years, and a primary diagnosis of GAD as obtained using the Mini Neuropsychiatric Interview for DSM-IV-TR. ${ }^{3}$ Patients were recruited from 42 outpatient treatment centers in 7 countries including the United States and parts of Europe. The mean age of study participants was 43.8 years of age, with $67.8 \%$ females. $^{3}$

Patients were screened including medical history and physical examination with $\mathrm{EKG}$, renal function tests, thyroid function tests, and urine drug screens. Patients were required to score at least 4 on the Clincical Global Impressions-Severity of Illness scale (CGI-SI). Patients were also required to score at least 10 on the Hospital Anxiety and Depression (HAD) anxiety subscale and at least 9 on the COVI anxiety scale. ${ }^{3}$

Unlike previous efficacy studies involving venlafaxine, the patients were evaluated using the HAM-A, but were not selected based on a minimum HAM-A score. Patients were also evaluated using the Sheehan Disability Scale (SDS). Response, remission and sustained improvement rates were also documented. ${ }^{3}$

Exclusion criteria for the study included a diagnosis of MDD (patients could not score $>3$ on any item of the Raskin Depression Scale) or substance use disorder within the past six months. Exclusion criteria also included a past year diagnosis of post-traumatic stress disorder, panic disorder, eating disorder, any prior history of a psychotic disorder, obsessive compulsive disorder, and bipolar disorder. Patients undergoing psychotherapy within 6 weeks of the study were also excluded from participation in the study. ${ }^{3}$ Patients were also excluded if they underwent 2 previous unresponsive trials of antidepressant or benzodiazepine medication for GAD. Patients were required to be free of all psychotropic medications for 2 to 4 weeks prior to randomization, depending on the psychotropic medication in question. ${ }^{3}$ Altogether 126 patients were excluded from the study based on these criteria. $^{3}$

All patients received single-blinded placebo treatment for 1 week before being randomized to receive placebo, $60 \mathrm{mg} /$ day of duloxetine, or $120 \mathrm{mg} /$ day of duloxetine. 
A patient's dose could be decreased to $30 \mathrm{mg} /$ day of duloxetine if they were unable to tolerate the starting dose. All patients who remained in the study were gradually increased to their randomly assigned dose over a two week period. The patients in the duloxetine groups were randomly assigned to either abrupt discontinuation or gradual ( 2 week) taper at the end of the study. ${ }^{3}$

The results of the first study yielded a statistically significant $(\mathrm{p}<0.001)$ improvement in HAM-A scores with duloxetine when compared to placebo (see Table 1). At $60 \mathrm{mg} /$ day of duloxetine the mean decrease in HAM-A score was 12.8 compared with only 8.4 for placebo. At $120 \mathrm{mg}$ /day the mean decrease in HAM-A score was $12.5 .^{3}$

Response was defined as $\geq 50 \%$ reduction in HAM-A at treatment endpoint. Sustained improvement was defined as a $30 \%$ reduction in HAM-A that was sustained at all subsequent visits. Remission was defined as a HAMA total score $\leq 7$ at completion of the study. ${ }^{3}$ Duloxetine demonstrated response rates of $58 \%$ at $60 \mathrm{mg} /$ day and $56 \%$ at $120 \mathrm{mg} /$ day compared to $31 \%$, for placebo $(\mathrm{p}<0.001)$. Remission rates were $31 \%$ at $60 \mathrm{mg} /$ day and $38 \%$ at $120 \mathrm{mg} /$ day compared with $19 \%$ for placebo ( $p<0.01$ and $p<0.001$ respectively). Sustained improvement rates were $63.6 \%$ at $60 \mathrm{mg} / \mathrm{day}$ and $66.9 \%$ at $120 \mathrm{mg} /$ day compared with $42.8 \%$ for placebo $(\mathrm{p}<0.001)$. Further, both duloxetine groups had greater improvements in Sheehan Disability Scale global scores ( $\mathrm{p}<0.001$ ), HAM-A psychic factor $(\mathrm{p}<0.001)$, and HAM-A somatic factor scores $(\mathrm{p}<0.01) .{ }^{3}$ Effect on overall disability will be discussed in more detail later.

The second and third studies were flexible-dose trials, also using mean change in HAM-A score to assess efficacy. ${ }^{13,14}$ The third study also provided a comparison to venlafaxine XR. ${ }^{13,14}$

The second study was a 10 week multi-center, double-blind, placebo-controlled trial. The study randomized 327 patients meeting DSM-IV-TR criteria for GAD to a duloxetine treatment group or to placebo (Table 1). The duloxetine arm received 60-120 mg of duloxetine with progressive titration and flexible dosing. ${ }^{13}$ The study involved 27 outpatient treatment centers. Patients were screened using the same CGI-Severity of Illness and HAD criteria as the first study. Patients were also required to score at least a 4 on the Covi Anxiety Scale. ${ }^{13}$ Exclusion criteria were essentially the same as the first study with 188 patients failing to meet the entry criteria. ${ }^{13}$

The study was single-blinded for the first week with all patients receiving placebo. Starting in week 2 , the study was double-blind and patients were randomly assigned to receive $60 \mathrm{mg}$ per day of duloxetine or placebo. Study visits were held at 1,2,4,7 and 10 weeks. A dosage decrease from $60 \mathrm{mg}$ to $30 \mathrm{mg}$ per day was allowed in the first 2 weeks, with all patients in the duloxetine arm required to take the $60 \mathrm{mg}$ dose by the end of the second week. The dose of duloxetine was increased if a patient's CGI score was $\geq 3$. The maximum dose was $120 \mathrm{mg} /$ day. $62 \%$ of the original sample completed the ten week therapy. ${ }^{13}$

The third study utilized the same inclusion and exclusion criteria as the second study. Besides that, third study also excluded patients meeting criteria for any Axis II disorder or having history of antisocial behavior. In the third study patients were randomized to duloxetine $(\mathrm{n}=162)$, venlafaxine $(\mathrm{n}=164)$, or placebo groups $(\mathrm{n}=161)($ Table 1$)$. Duloxetine was started at a dose of $30 \mathrm{mg}$ per day for 1 week and was then increased to a range of 60 to $120 \mathrm{mg}$ per day thereafter. The dosage increases for duloxetine followed the same algorithm as the second study following the second week of treatment, with a maximum dosage of $120 \mathrm{mg}$ per day. ${ }^{14}$

Venlafaxine was utilized in the XR formulation and was started at $37.5 \mathrm{mg}$ per day and increased to a dosage range of 75 to $225 \mathrm{mg}$ per day. According to Eli Lilly the study was not "powered to compare efficacy between Cymbalta and Effexor XR." ${ }^{\prime 8}$ Eli Lilly states that data from another study will be pooled with data from this study to provide a more statistically powerful head-to-head comparison of duloxetine and venlafaxine XR. ${ }^{8}$

Results of both study 2 and study 3 showed a statistically significant decrease in HAM-A scores for the duloxetine

Table I Efficacy studies of duloxetine in the treatment of GAD

\begin{tabular}{lllllllll}
\hline Study & $\begin{array}{l}\text { Number } \\
\text { of subjects }\end{array}$ & $\begin{array}{l}\text { Average } \\
\text { age }\end{array}$ & Gender & Study length & Design & Dosing & Change in HAM-A & $\begin{array}{l}\text { Statistical } \\
\text { significance }\end{array}$ \\
\hline Koponen et al $^{3}$ & 513 & 43.8 & $67 \%+$ & 9 week & Placebo-controlled & Fixed & $\downarrow 12.8(60 \mathrm{mg} /$ day $)$ & $\mathrm{P}<0.00 \mathrm{I}$ \\
Rynn et al $^{13}$ & 327 & 41.6 & $61.7 \%+$ & 10 week & Placebo-controlled & Flexible & $\downarrow 8.12$ & $\mathrm{P}=0.023$ \\
Hartford et al $^{14}$ & $487^{\mathrm{a}}$ & $40.4^{\mathrm{b}}$ & $64.2 \%$ \% & 10 week & Placebo-controlled & Flexible & $\downarrow 11.8$ & $\mathrm{P} \leq 0.00 \mathrm{I}$ \\
\hline
\end{tabular}

aduloxetine efficacy study with venlafaxine XR comparator arm [duloxetine $(n=162)$, venlafaxine $(n=164)$, and placebo groups $(n=161)$ ]

bduloxetine group. 
groups when compared to placebo ( $\mathrm{p}=0.023$ for study 2 and $\mathrm{p}<0.01$ for study 3$){ }^{13,14}$

Secondary efficacy measures for study 2 showed statistically significant improvement in HAM-A items for anxious mood $(-0.98, \mathrm{p}=0.006)$, tension $(-0.98, \mathrm{p}=0.014)$, fears $(-0.49, \mathrm{p}=0.049)$, cognitive $(-0.98,<0.001)$, depressed $\operatorname{mood}(-0.43, \mathrm{p}=0.003)$, and behavior $(-0.66, \mathrm{p}=0.011)$ compared with placebo. ${ }^{13}$ In the second study the CGI-I endpoint (2.68) and PGI-I endpoint (2.88) also showed a statistically significant change with respect to placebo $\left(p=0.04\right.$ and $p=0.046$ respectively) ${ }^{13}$ The HAM-A psychic factor scale also showed statistically significant improvement $(-5.33, \mathrm{p}<0.001)$ compared with placebo in the second study. ${ }^{13}$ The HADS anxiety subscale ( -3.92 , $\mathrm{p}=0.001)$ also showed a statistically significant change when compared to placebo in the second study. ${ }^{13}$ The HAM-A somatic factor, insomnia, somatic muscular, somatic sensory, cardiovascular, respiratory, gastrointestinal, genitourinary, and autonomic items did not show a statistically significant change when compared to placebo. ${ }^{13}$ The HADS depression subscale also did not show findings that were statistically significant. ${ }^{13}$

Secondary efficacy measures for study 3 showed significant improvement when compared to placebo (in the duloxetine group) for the HAM-A psychic factor (-7.01), HAM-A anxious mood (-1.40), HAM-A tension (-1.37), CGI-I endpoint (2.39), PGI-I endpoint (2.60), HADS anxiety subscale (-6.22) and HADS depression subscale $(-3.91){ }^{14}$

The above studies showed duloxetine to be an effective treatment for GAD. However, it is often difficult to extrapolate clinical trial data to "real world" patients encountered in clinical practice.

Tunis et al defined a practical clinical trial as "a study design that compares clinically important interventions, a diverse population of study participants representative of clinical practice, inclusion of a range of heterogeneous practice settings that are also representative of clinical practice, and measurement of a broad range of clinically relevant health outcomes. ${ }^{20}$ Strengths of the above studies include use of standardized rating scales such as HAM-A in the evaluation of patients, large sample-size, multi-center, effective randomization, and double-blind design.

However, generalizability of research findings from clinical trials is a methodological concern. Because most of the studies exclude many participants with general medical and psychiatric co-morbidities, generalizability of the results is limited. The study also excluded patients with a history of previous unresponsiveness to SSRI or benzodiazepine in the treatment of GAD, therefore selecting out non-responders who may be encountered in clinical practice. Homogeneity between the sample population and the target population is necessary so that the results can be extrapolated. Other limitations of the above studies include a short time to evaluate treatment and response. Patients in clinical practice typically utilize antidepressant medications for longer than 9 to 10 weeks.

Results of long-term studies could provide information on remission rates that would be clinically useful. Long-term studies would also provide clinicians with information on maintenance treatment. Davidson, et al published results of a 26 week double-blind, placebo controlled, trials looking at efficacy of duloxetine in relapse prevention. Study patients first completed a 26-week, open-label, acute-therapy phase. Patients included in the study were those that responded to this acute phase treatment, defined as $\geq 50 \%$ reduction in Hamilton Anxiety Rating Scale and "much" to "very much" improved ratings for the last two visits of acute open label treatment. For study purposes, relapse was defined as $\geq 2$ point increase in illness severity ratings or by discontinuation for lack of efficacy. $41.8 \%$ of placebo-treated patients experienced relapse of their illness based on the above criteria, compared with only $13.7 \%$ of duloxetine treated patients $(p \leq 0.001)$ at the end of the second 26 weeks (continuation phase). ${ }^{21}$

Pollack, et al pooled data from three clinical trials $(n=688)$ and showed that $100 \%$ of responders to duloxetine $(>80 \%$ improvement of HAM-A) at week 2 and $93 \%$ of responders at week 4 were responders to treatment at endpoint. Similar data is not available for SSRI's at this time, but would be helpful in evaluating treatment options clinically.22

We have now reviewed the efficacy data that lead to FDA approval for treatment of GAD. Data from these studies was also pooled to examine improvement in quality of life.

\section{Quality of life}

In clinical practice, improvement of an individual's qualify of life is a sought-after result that is difficult to pull from research studies. A 2008 study by Pollack et al pooled data from 3 studies to assess if duloxetine improved quality of life in patients meeting DSM-IV-TR criteria for GAD. ${ }^{23}$ By utilizing comparative norms, the analysis examined the clinical importance of the functional outcomes improvements reported in the above studies. Specifically, the study sought to examine how this data on functional outcomes would translate into real-world clinical improvement. ${ }^{23}$

Individual patient data was pooled from the above studies, with outcome criteria defined as above. At baseline, $89 \%$ of patients were impaired on the Sheehan Disability Scale (SDS) 
SDS Score at Endpoint

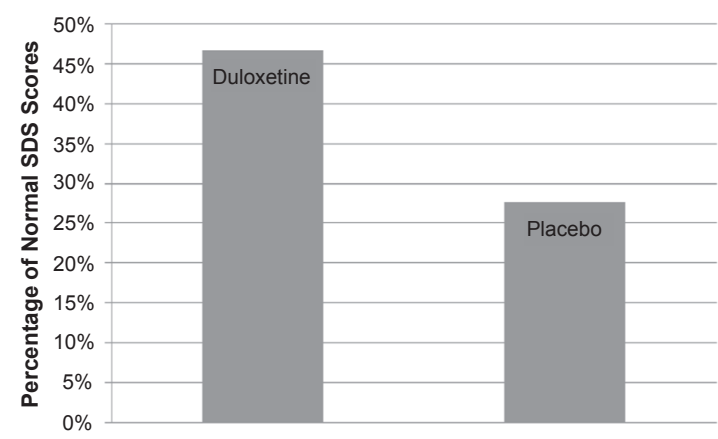

Figure I Quality of life data in patients with GAD treated with duloxetine.

global functioning and $95 \%$ of patients were impaired on the Quality of Life Enjoyment and Satisfaction Questionnaire Short Form (Q-LES-Q-SF). ${ }^{23}$ The study confirmed the belief that patients with GAD have impaired functioning and quality of life. Following treatment, the duloxetine group showed statistically significant improvement when compared to placebo $(\mathrm{p} \leq 0.001) .{ }^{23}$ The mean SDS score for the duloxetine treatment groups at completion ranged from 7.4 to 9.5 compared with a mean range of 11.4 to 12.1 for placebo-treated groups. ${ }^{23} 47 \%$ of duloxetine treated patients achieved a normative SDS score at studies end, compared with $28 \%$ of placebo-treated patients (Figure 1). ${ }^{23}$

Duloxetine treated patients not only demonstrated improved quality of life, but improved overall satisfaction as studied by the Q-LES-Q-SF. ${ }^{4}$ Mean endpoint scores on the Q-LES-Q-SF ranged from $65.9 \%$ to $67.9 \%$ for the duloxetine group compared to $58.5 \%$ to $60.9 \%$ for the placebo group (Figure 1 ). ${ }^{23}$ Two of the three clinical trials demonstrated a significant improvement of Q-LES-Q-SF compared with placebo. Duloxetine treated patients were not only able to better carry out role functioning, but were more satisfied with their quality of life. ${ }^{24,25}$

Over 1100 patients were included in this analysis with the improvements in quality of life being replicated in all three. While such data is useful in real-world clinical practice, studies with longer follow-up would better determine long-term maintenance of such improvements. ${ }^{24}$

TCAs and venlafaxine are often utilized clinically in patients with concomitant chronic pain issues. The mechanism of action of duloxetine lends itself to similar use, and we will examine data in this regard.

\section{Chronic pain}

Duloxetine received FDA approval for the treatment of diabetic peripheral neuropathic pain in 2004 and is often

\section{$\bar{Q}-\bar{L} \bar{E} \bar{S}-\bar{Q}-\bar{S} \bar{F}$ Score at Endpoint}

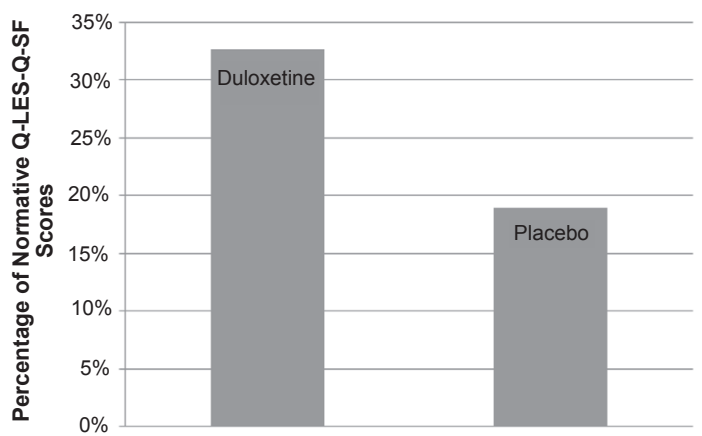

utilized clinically in patients whose anxiety is marked by somatic symptoms or who have concomitant chronic pain. Pooled results from two studies showed that $44.4 \%$ of patients identified with GAD had visual analog scale (VAS) scores $\geq 30$. $^{26}$

Hartford et al conducted a post hoc evaluation of two double-blind, placebo-controlled trials examining efficacy of duloxetine in GAD. Duloxetine showed reduction in pain on all 6 VAS pain scales $(p<0.001) .{ }^{27}$ Russell et al also pooled data from two double-blind, placebo-controlled trials looking at efficacy of duloxetine in GAD. Results of this pooled analysis also showed that patients receiving duloxetine showed improvement on all 6 VAS pain scales ( $p<0.001$ for overall pain, $p=0.009$ for headache, $p=0.003$ for back pain, $\mathrm{p}<0.001$ for shoulder pain, $\mathrm{p}<0.001$ for daily interference due to pain, and $\mathrm{p}<0.001$ for proportion of the day while awake with pain). ${ }^{26}$

As above this is of particular interest to clinicians because of the frequent overlap between pain and anxiety in GAD. Also, as further advances in neuroscience come to light, further structural and neurophysiologic links between pain and anxiety in GAD could be uncovered.

Now that we have evaluated data that is pertinent to clinical practice for duloxetine, we will take a brief look at the data for venlafaxine XR, another SNRI that is FDA approved for treatment of GAD.

\section{Venlafaxine XR}

While no head-to-head trials comparing duloxetine with other medications for the treatment of GAD currently exist, venlafaxine XR, an SNRI, was FDA approved for the treatment of GAD in 1991.

In 2000, a study published in the American Journal of Psychiatry examined the efficacy of venlafaxine XR in 
the treatment of non-depressed outpatients with GAD. ${ }^{28}$ The study was an 8-week, double-blind, fixed dose trial that randomized patients to placebo $(n=96)$ or venlafaxine $X R .^{28}$ Venlafaxine XR was dosed at $75 \mathrm{mg} /$ day $(\mathrm{n}=86), 150 \mathrm{mg} /$ day $(\mathrm{n}=81)$, or $225 \mathrm{mg} /$ day $(\mathrm{n}=86) .{ }^{28}$

Patients included in the study scored at least an 18 on the HAM-A and at least a 2 on the anxious mood and tension factors subscale. Unlike the above studies involving duloxetine, this study utilized HAM-A score as part of the inclusions criteria. Primary efficacy measures included change in total HAM-A score (Table 2).

In 2000, results of a 6-month, double blind, placebo-controlled trial showed long-term effectiveness in the use of venlafaxine XR to treat GAD. ${ }^{29}$ The study was a 28 -week, double-blind, placebo-controlled, flexible dose trial involving 251 nondepressed adult outpatients meeting DSM-IV criteria for GAD (Table 2). ${ }^{29}$

The goal of this article is not to compare venlafaxine XR to duloxetine for the treatment of GAD. However, clinicians may often need to choose between these two medications as an initial therapy or as an option for patients who have failed a trial of a different medication.

The two studies that we discussed on venlafaxine XR used HAM-A score as an entry criteria for the study, while the studies we discussed on duloxetine did not. By not using HAM-A score as an entry criteria, the duloxetine study designs may have reduced the potential for rater bias. $^{14}$

An additional study comparing venlafaxine XR with duloxetine will generate data that will be pooled with the Hartford study to provide a better powered comparison of the two agents. ${ }^{14}$

At this time venlafaxine XR and duloxetine are both safe and efficacious treatment options for clinicians to consider in GAD. Similar to our discussion of duloxetine as a possibility for patients with somatic manifestations of anxiety, choosing venlafaxine XR may often be based on co-morbidities. Additional studies in this area could also be useful to clinicians.

\section{Future studies}

An NIMH sponsored trial (NCT00183274) is currently recruiting subjects to study "Effectiveness of Long-Term Versus Short-Term Treatment of Generalized Anxiety Disorder With Venlafaxine XR." 30 The National Taiwan Hospital (NCT00491348) is currently recruiting subjects to study "Effects of Duloxetine on Pathological Worry in Patients with Generalized Anxiety Disorder: A fMRI Study." ${ }^{30}$

\section{Conclusion}

Duloxetine is an FDA approved treatment option for GAD that has been studied in acute phase 9- to 10-week doubleblind, placebo-controlled clinical trials. Duloxetine has also been evaluated for relapse prevention (26-week continuation) in patients whose GAD symptoms have responded after 26 weeks of acute phase treatment. Duloxetine may also be particularly useful to clinicians treating GAD patients with concomitant chronic pain or who have significant somatic symptoms as a part of their anxiety.

The information presented is important because it forms the basis for the size and quality of the systematic database upon which physicians can form evidence based opinions about the reliability, safety, tolerability and efficacy of duloxetine in patients with GAD. This article will help physicians to interpret clinical studies properly and apply that knowledge to their patients. As the evidence base with regard to maintenance therapy and treatment of specific symptoms of GAD grows, clinicians will be able to make an informed decision about which patients are the most appropriate candidates for a trial of duloxetine.

\section{Disclosures}

Dr Khan is on the speaker panel for Wyeth pharmaceuticals. Dr Macaluso has no disclosures.

Table 2 Efficacy studies of venlafaxine $X R$ in the treatment of GAD

\begin{tabular}{|c|c|c|c|c|c|c|c|c|}
\hline Study & $\begin{array}{l}\text { Number } \\
\text { of subjects }\end{array}$ & Average age & Gender & Study length & Design & Dosing & Change in HAM-A & $\begin{array}{l}\text { Statistical } \\
\text { significance }\end{array}$ \\
\hline Rickels et $\mathrm{al}^{28}$ & 349 & $39.6^{\mathrm{a}}$ & $56 \% \mathrm{a}^{\mathrm{a}}+$ & 8 weeks & Placebo-controlled & Fixed & $\downarrow / 2.36(150 \mathrm{mg} /$ day $)$ & $P=0.07$ \\
\hline Gelenberg et $\mathrm{a}^{29}$ & 251 & $4 I^{* *}$ & $68 \%$ b 9 & 6 months & Placebo-controlled & Flexible & $\downarrow 13.4$ & $\mathrm{P}<0.00 \mathrm{I}$ \\
\hline Hartford et al ${ }^{14}$ & $487^{c}$ & $40.1^{b}$ & $62.2 \% \mathrm{~b}+$ & 10 weeks & Placebo-controlled & Flexible & $\downarrow 12.4$ & $P \leq 0.01$ \\
\hline
\end{tabular}

*venlafaxine group randomized to $150 \mathrm{mg} /$ day.

${ }^{b}$ venlafaxine group.

'duloxetine efficacy study with venlafaxine XR comparator arm [duloxetine $(n=162)$, venlafaxine $(n=164)$, and placebo groups $(n=161)$ ]. 


\section{References}

1. Greenberg PE, Sisitsky T, Kessler RC, et al. The economic burden of anxiety disorders in the 1990s. J Clin Psychiatry. 1999;60(7):427-435.

2. American Psychiatric Association Diagnostic and Statistical Manual of Mental Disorders. 4th ed., text revision. Washington DC: American Psychiatric Press, Inc; 2000.

3. Koponen H, Allgulander C. Efficacy of duloxetine for the treatment of generalized anxiety disorder: implications for primary care physicians. Prim Care Comp J Clin Psychiatry. 2007;9(2):100-107.

4. Wittchen H-U, Hoyner J. Generalized anxiety disorder: nature and course. J Clin Psychiatry. 2001;62(suppl 1)1:15-10.

5. Butler G, Fennel M, Robson P, Gelder M. Comparison of behavior therapy and cognitive behavior therapy in the treatment of generalized anxiety disorder. J Consult Clin Psychol. 1991;59:167-175.

6. Preskorn SH. Milnacipran: A dual norepinephrine and serotonin reuptake pump inhibitor. J Psychiatr Pract. 2004;10:119-126.

7. Eli Lilly. Duloxetine package insert, September 2004. Available at www.insidecymbalta.com.

8. Eli Lilly [online]: www.lilly.com.

9. Preskorn SH. Duloxetine. J Psychiatr Pract. 2004;10(6):375-385.

10. Gupta S, Nihalani N. Duloxetine: review of its pharmacology, and therapeutic use in depression and other psychiatric disorders. Ann Clin Psychiatry. 2007;19(2):125-132.

11. Skinner MH, Kuan HY, Sathirakul K, et al. Duloxetine is both an inhibitor and a substrate of cytochrome P4502D6 in healthy volunteers. Clin Pharmacol Ther. 2003;73:170-177.

12. Physicians Desk Reference. 62nd ed. Montrale, NJ: Thomson Healthcare Inc.; 2008. p. 1791-1798.

13. Rynn M, Russell J, Erickson J, et al. Efficacy and safety of duloxetine in the treatment of generalized anxiety disorder: a flexible dose, progressive titration, placebo-controlled trial. Depress Anxiety. 2008;25(3):182-189.

14. Hartford J, Kornstein S, Liebowitz M, et al. Duloxetine as an SNRI treatment for generalized anxiety disorder: results from a placebo and active-controlled trial. Int Clin Psychopharm. 2007;22:167-174.

15. Sharma A, Goldberg MJ, Cerimele BJ. Pharmacokinetics and safety of duloxetine, a dual-serotonin and norepinephrine reuptake inhibitor. J Clin Pharmacol. 2000;40:161-167.

16. Davidson J, Allgulander C. Efficacy and tolerability of duloxetine in elderly patients with generalized anxiety disorder: a pooled analysis of four randomized, double-blind, placebo-controlled studies. Hum Psychopharmacol Clin Exp. 2008;23:519-526.
17. Lantz RJ, Gillespie TA, Rash TJ, et al. Metabolism, excretion, and pharmacokinetics of duloxetine in healthy human subjects. Drug Metab Dispos. 2003;31:1142-1150.

18. Karpa KD, Cavanaugh JE, Lakoski JM. Duloxetine pharmacology: Profile of a dual monoamine modulator. CNS Drug Rev. 2002;8:361-376.

19. Parikh AR, Thatcher BT, Tamano EA, Liskow BI. Suicidal ideation associated with duloxetine use: a case series. J Clin Psychopharm. 2008;28(1):101-102.

20. Tunis SR, Stryer DB, Clancy CM. Practical clinical trials: increasing the value of clinical research for decision making in clinical and health policy. JAMA. 2003;290:1624-1632.

21. Davidson J, Wittchen HU. Duloxetine treatment for relapse prevention in adults with generalized anxiety disorder: A double-blind placebo-controlled trial. J Euro Neuro. 2008;18:673-681.

22. Pollack MH, Kornstein SG, Spann ME, Crits-Christoph P, Raskin J, Russell JM. Early improvement during duloxetine treatment of generalized. J Psychiatr Res. 2008;42(14):1176-1184.

23. Pollack MH, et al. Examining quality of life in patient's with generalized anxiety disorder. J Psychiatr Res. 2008; doi: 10.1016/ j.jpsychires. 2007.11.006.

24. Kessler RC, DuPont RL, Berglund P, et al. Impairment in pure and comorbid generalized anxiety disorder and major depression at 12 months in two national surveys. Am J Psychiatry. 1999;156:1915-1923.

25. Endicott J, Russell JM, Raskin J, et al. Duloxetine treatment for role functioning improvement in generalized anxiety disorder: three independent studies. J Clin Psychiatry. 2007;68(4):518-524.

26. Russell J, Weisberg R. Efficacy of duloxetine in the treatment of generalized anxiety disorder in patients with clinically significant pain symptoms. Depress Anxiety. 2008;25:E1-E11.

27. Hartford J, Endicott J. Implications of pain in generalized anxiety disorder: efficacy of duloxetine. Prim Care Companion J Clin Psychiatry. 2008;10(3).

28. Rickels K, Pollack MH, Sheehan DV, Haskins JT. Efficacy of extendedrelease venlafaxine in nondepressed outpatients with generalized anxiety disorder. Am J Psych. 2000;157:968-974.

29. Gelenberg A, Lydiard RB, Rudolph RL, Aquiar L, Haskins JT, Salinas E. Efficacy of venlafaxine extended-release capsules in nondepressed outpatients with generalized anxiety disorder: a 6 month randomized controlled trial. JAMA. 2000;283(23):3082-3088.

30. US Institute of Health, Clinical Trials Registry. Available from www. clinicaltrials.gov. 
